\title{
HIGH-RESOLUTION IMAGING OF TEXTURE AND MICROSTRUCTURE BY THE MOVING DETECTOR METHOD
}

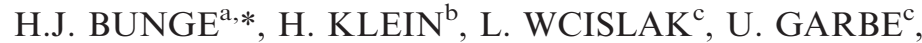 \\ W. WEI $\beta^{\mathrm{d}}$ and J.R. SCHNEIDER ${ }^{\mathrm{c}}$ \\ ${ }^{a}$ Department of Physics and Physical Technologies, Technical University of Clausthal, \\ Leibnizstr. 4, D-38678 Clausthal-Zellerfeld, Germany; ${ }^{\mathrm{b}}$ Department of Crystallography, \\ University of Göttingen, Goldschmidtstr. 1, D-37077 Göttingen, Germany; ${ }^{\mathrm{C}} H A S Y L A B$ at \\ Deutsches Elektronen Synchrotron DESY, Notkestr. 85, D-22603 Hamburg, Germany; \\ ${ }^{\mathrm{d}}$ Gerstenkamp 9, D-51061 Köln, Germany
}

(Received 13 September 2003)

\begin{abstract}
In order to describe texture and microstructure of a polycrystalline material completely, crystal orientation $\mathbf{g}=\left\{\varphi_{1} \Phi \varphi_{2}\right\}$ must be known in all points $\mathbf{x}=\left\{x_{1} x_{2} x_{3}\right\}$ of the material. This can be achieved by locationresolved diffraction of high-energy, i.e. short-wave, X-rays from synchrotron sources. Highest resolution in the orientation- as well as the location-coordinates can be achieved by three variants of a detector "sweeping" technique in which an area detector is continuously moved during exposure. This technique results in two-dimensionally continuous images which are sections and projections of the six-dimensional "orientation-location" space. Further evaluation of these images depends on whether individual grains are resolved in them or not. Because of the high penetration depth of high-energy synchrotron radiation in matter, this technique is also, and particularly, suitable for the investigation of the interior of big samples.
\end{abstract}

Keywords: High-energy synchrotron radiation; High orientation resolution; High location resolution; Local textures; Grain-resolved structures; $\gamma \rightarrow \alpha$ transformation; Fe-Ni meteorite; Orientation relationship

\section{INTRODUCTION}

The texture of a polycrystalline material is defined, in the classical sense, by the volume fraction of crystallites with the orientation $\mathbf{g}=\left\{\varphi_{1} \Phi \varphi_{2}\right\}$. This definition does not include the microstructure, i.e. the positions of the grains in the material. The complete description of a polycrystalline aggregate is given by the "orientation-location" function $\mathbf{g}(\mathbf{x})$ which specifies the orientation $\mathbf{g}$ in any (small) volume element at the position $\mathbf{x}=\left\{x_{1} x_{2} x_{3}\right\}$ in the material. Hence, it is necessary to "image" the complete six-dimensional "orientation-location" space $\left\{\varphi_{1} \Phi \varphi_{2} x_{1} x_{2} x_{3}\right\}$. This can be done by location-resolved diffraction methods. Among these, the EBSD-technique (Electron Back Scattering Diagrams) with automated location scanning is well known (e.g., the

*Corresponding author. E-mail: Hans.Bunge@t-online.de 
OIM (Adams et al., 1994) or ACOM (Schwarzer, 1997) technique). Because of the low pentration depth of electrons in materials, this technique "sees", however, only a very thin layer of the material. On the other hand, high-energy synchrotron radiation with wavelengths in the order of $\sim 0.1 \AA$ or $\sim 100 \mathrm{keV}$ photon energy has penetration depths in the centimeter range. At the same time its high parallelity allows extremely high orientation resolution (better than that of electron diffraction). The high intensity of synchrotron radiation allows the use of very narrow incident beams and thus a high lateral resolution. Hence, it is possible to obtain images of the six-dimensional orientation-location space with high orientation- as well as location-resolution.

\section{THE EXPERIMENTAL SET-UP}

The investigations were done with the multi-purpose diffraction installation at the beam line BW5 at HASYLAB/DESY in Hamburg, shown schematically in Fig. 1. A monochromator selects a monochromatic beam out of the white spectrum. This beam is diffracted in the polycrystalline sample and the diffraction diagram is registered in an area detector, actually an image plate of $345 \mathrm{~mm}$ diameter at $\sim 1 \mathrm{~m}$ from the sample (Wcislak et al., 2002). Because of the small diffraction angles in the order of $<\sim 5^{\circ}$ the detector catches virtually the whole diffraction diagram. Additionally to this basic configuration of the instrument two different slit systems can be installed alternatively between sample and detector, i.e. a diffraction angle slit and a diffraction plane slit. Furthermore, the detector is being continuously translated during exposure (perpendicular to the incident beam in the drawing plane of Fig. 1).

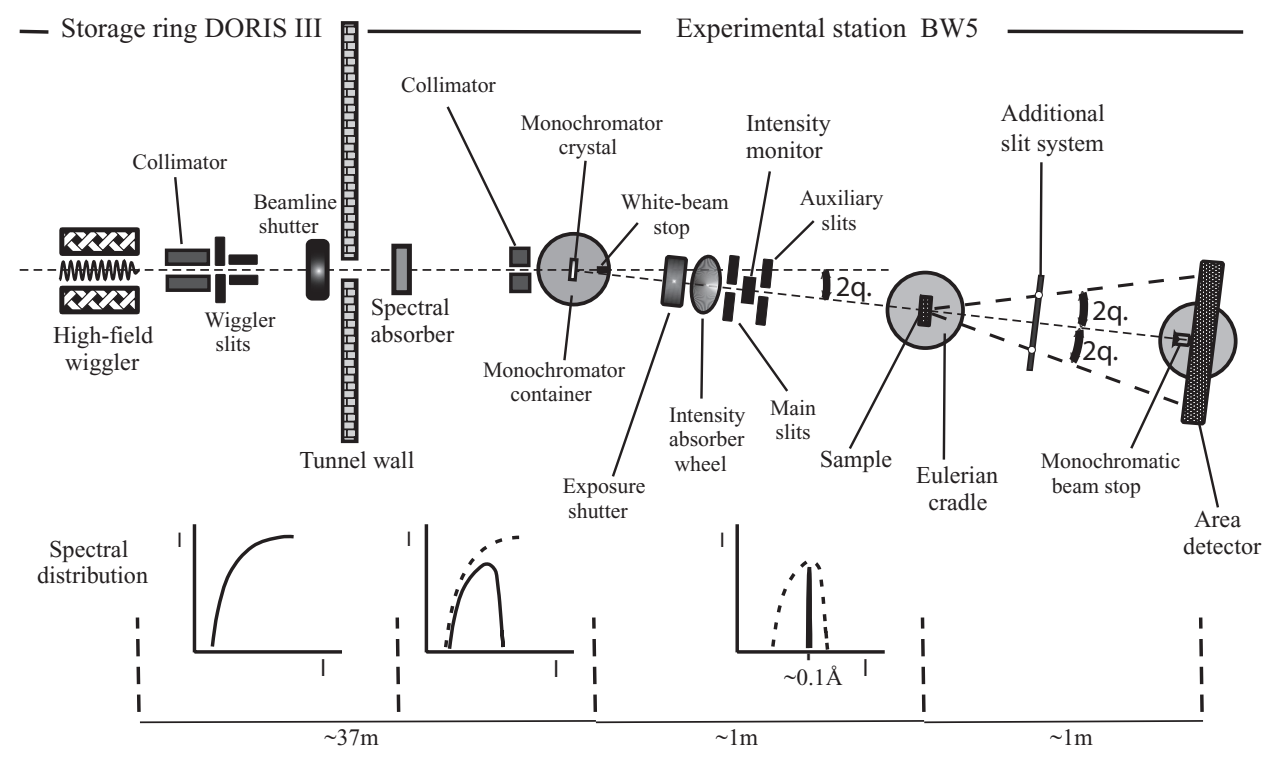

FIGURE 1 The diffraction installation at the beamline BW5 at the storage ring DORIS in HASYLAB/ DESY in Hamburg. 


\subsection{Measurement with the Diffraction Angle Slit}

The diffraction angle slit system is shown schematically in Fig. 2. It allows only a sector (smaller than $180^{\circ}$ ) of one selected Debye-Scherrer circle to reach the detector. During exposure the detector is being translated in $y$-direction, perpendicular to the incident beam. At the same time the sample can either be rotated (e.g. around the axis $\omega$, technique $(a)$ ) or translated (e.g. in $y$-direction, technique $(b)$ ) with speeds adapted to the translation speed of the detector.

\subsubsection{Pole Figure Measurement}

In the first case (technique $(a)$ ) crystallites, the $(h k l)$ planes of which are in reflection orientation at the angles $\{\omega \gamma\}$, are imaged. This is essentially the $(h k l)$-pole figure, though expressed in terms of $\{\omega \gamma\}$ instead of the conventional representation in terms of the angles $\{\alpha \beta\}$ (Wcislak et al., 2002).

\subsubsection{Orientation-specific Location Diagrams and Local Textures}

In the second case (technique $(b)$ ) crystallites with the angles $\gamma$ (at fixed $\omega$ ) are imaged as a function of their position $y$ in the sample (Bunge et al., 2002).

In the case of grain-resolved textures (shown later in Fig. 5, right half) one thus sees the positions (coordinates $y$ ) of individual grains with the orientation coordinates $\omega$ (fixed) and $\gamma$.

In the case of continuous textures (Fig. 5, left part) each point in the diagram represents the pole density of all crystallites with the orientations $\{\omega \gamma\}$ as a function of their position (coordinate $y$ ). This contains information of the local texture (though not explicitly).

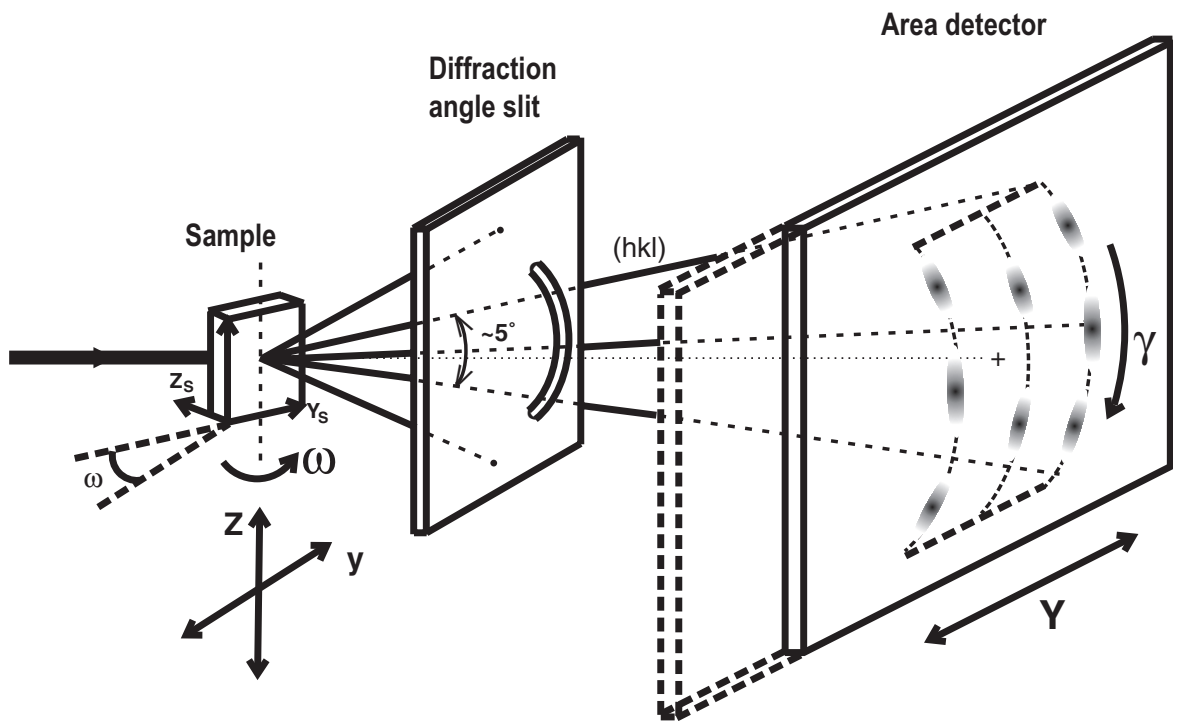

FIGURE 2 The diffraction angle slit used together with a moving area detector (schematic) (techniques $(a)$ and $(b))$. 


\subsection{Measurement with the Diffraction Plane Slit}

The diffraction plane slit system is shown in Fig. 3(a). In this case the incident beam has the form of a thin band extended in $z$-direction. The entrance slit and the diffraction plane slit fix together the diffraction plane. Crystallites in reflection orientation (with different $(h k l)$ ) reflect the incident beam as is shown in detail (for one $(h k l)$ ) in Fig. 3(b). During exposure both, the sample and the detector, are continuously translated in $y$-direction. This way, the projected cross-sections of grains in reflection orientation are imaged on the detector (technique (c)) (Bunge et al., 2003). In contrast to technique $(b)$ any one of the two-dimensional images, seen in Fig. 3(a), shows only grains with one fixed diffraction vector, these, however, as a function of two location coordinates $y$ and $z$.

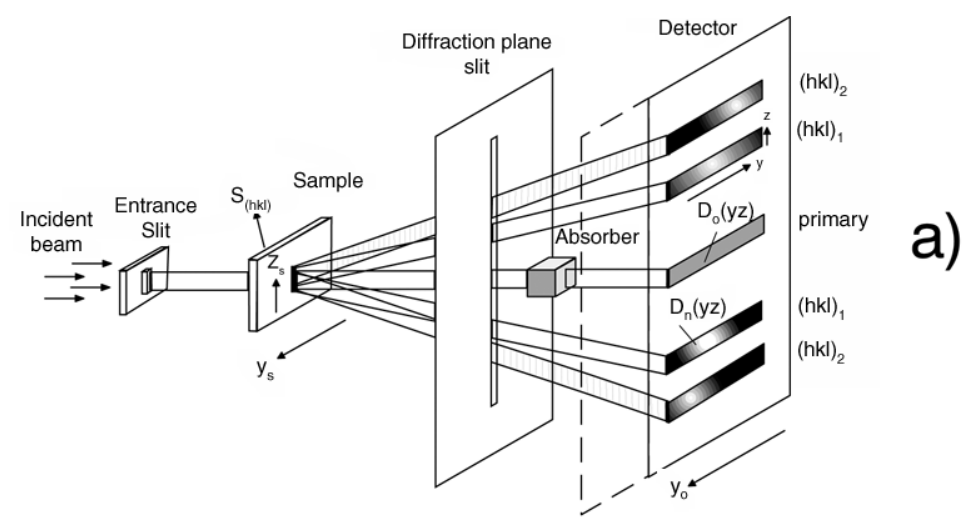

$\begin{array}{cll}\begin{array}{c}\text { Entrance } \\ \text { Slit }\end{array} & \text { Sample } & \begin{array}{l}\text { Diffraction } \\ \text { plane split }\end{array}\end{array}$

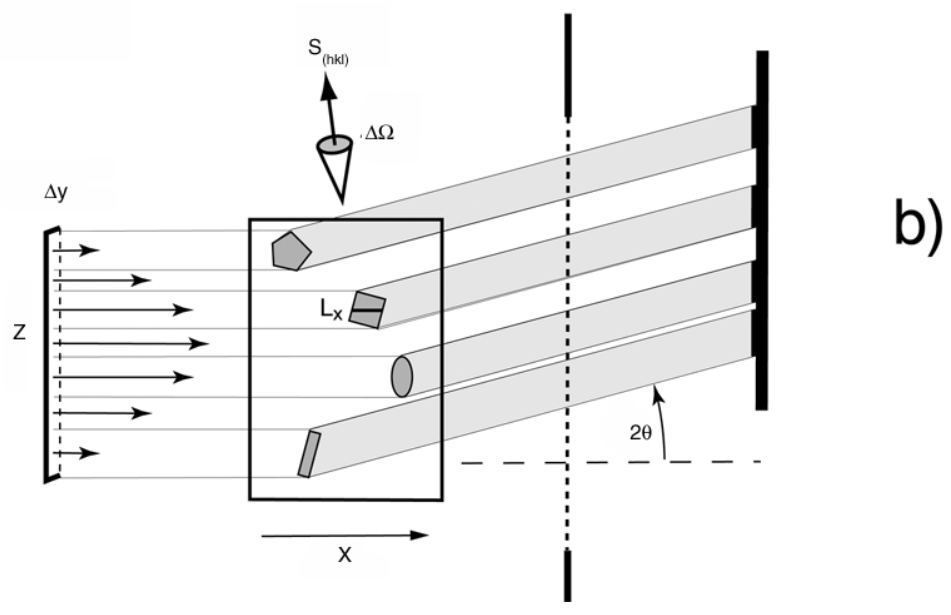

FIGURE 3 The diffraction plane slit system used together with a moving area detector (technique $c$ ): (a) the slit system (schematic); (b) reflection of X-rays in crystals which are in reflection orientation with the specified diffraction vector $\mathbf{S}$ (within a tolerance $\Delta \Omega$ ). 
a)

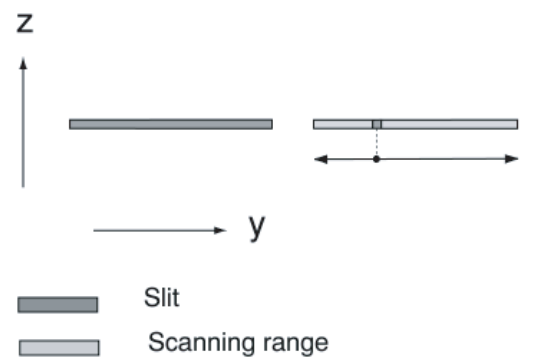

c)

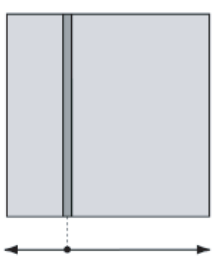

FIGURE 4 Slit sizes and sweeping ranges for the techniques $(a),(b)$ and $(c)$, dark grey: slit size, light grey : sweeping range.

\subsection{Slit Sizes for the Three Methods}

The three methods $(a),(b)$ and $(c)$ require different shapes and sizes of the entrance slits as is shown in Fig. 4. Hence, method (a) has no resolution in $y$-direction. Method (b) has resolution in $y$ - and $z$-direction (both given by the slit sizes) which may, however, be different in both directions. In method $(c)$, resolution in $y$-direction is given by the slit size, and in $z$-direction it is given by the parallelity of the incident and reflected beam. The scanning range in $z$-direction is given by the length of the slit in $z$-direction, which, in turn, is limited by the basic parameters of the beamline. The scanning ranges of the methods $(b)$ and $(c)$ in $y$-direction are, in principle, not limited. However, they determine the imaging scale in this direction onto the detector. In order to reach a good scaling factor and hence a good location resolution the scanning range in $y$-direction must be adapted to the detector diameter in $y$-direction (Bunge et al., 2003a).

All measurements, shown in this article, were carried out with the routine entrance slits of the diffraction instrument BW5 shown in Fig. 1. These slits can be reliably used with openings $\geq 0.1 \mathrm{~mm}$. Hence, location resolution of the shown diagrams of types $(b)$ and $(c)$ are in this order of magnitude.

However, other special slit systems are also available which allow openings, and hence location resolution, in the order of $10 \mu \mathrm{m}$ or lower. Hence, location resolution in this order of magnitude may be obtained with the shown techniques.

\section{RESULTS}

Examples of images obtained with the three techniques $(a),(b)$ and $(c)$ are shown in Fig. 5. They must be distinguished according to whether individual grains are resolved or not, as is shown on the right and left part of this figure, respectively.

Figure 6 summarizes the imaging conditions of the three techniques. Each of them images two of the six coordinates $\{\omega \gamma \psi x y z\}$ continuously, other coordinates have fixed (but selectable) values, and some of the coordinates cannot be distinguished (i.e. they are projection directions). These are particularly a rotation $\psi$ around the normal direction of the reflecting lattice plane $(h k l)$, i.e. around the diffraction vector, and the direction $x$ in the sample along the incident beam. 


\section{Continuous}

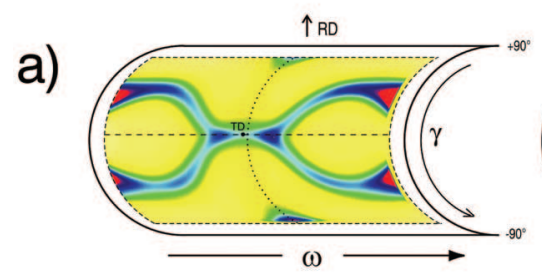

b)

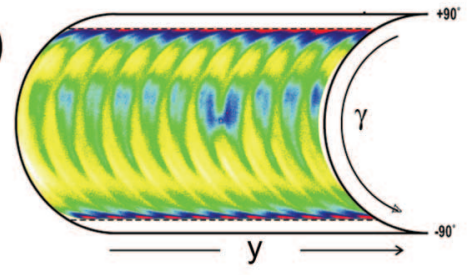

c)

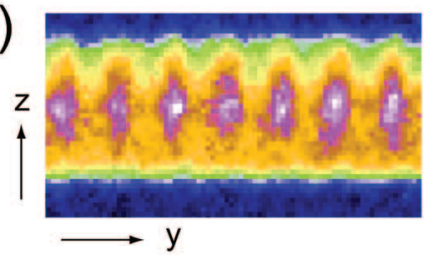

\section{Grain-resolved}
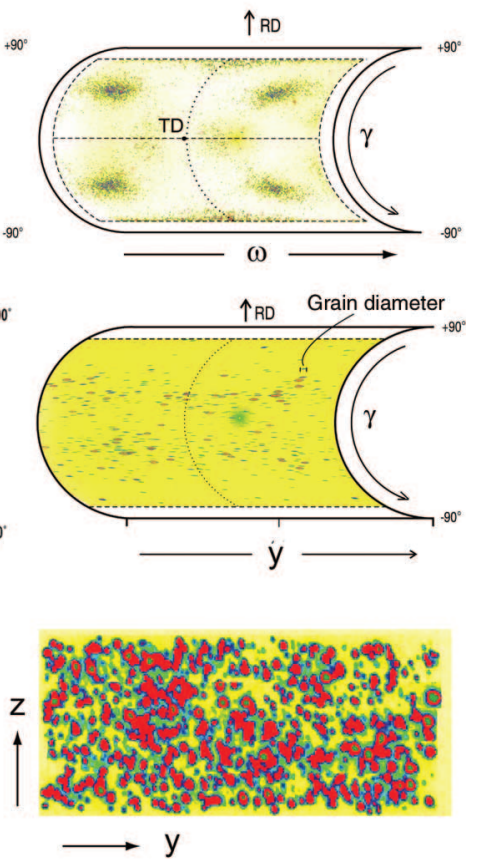

FIGURE 5 Example diagrams obtained with the techniques $(a),(b)$ and $(c)$. Left side: continuous textures, (a) cold rolled nickel sheet, (b), (c) lamellar copper tube. Right side: grain-resolved textures, (a), (b), (c) recrystallized nickel sheet.

\begin{tabular}{|c|c|c|c|c|c|c|}
\hline \multirow{2}{*}{ Method } & \multicolumn{3}{|c|}{ Orientation } & \multicolumn{3}{|c|}{ Location } \\
\hline & $\omega$ & $\gamma$ & $\psi$ & X & Y & Z \\
\hline a & $\longleftrightarrow$ & $\longleftrightarrow$ & $\square$ & & & \\
\hline b & 0 & $\longleftrightarrow$ & $\square$ & $\square$ & $\longleftrightarrow$ & 0 \\
\hline c & 0 & 0 & $\square$ & & & \\
\hline
\end{tabular}

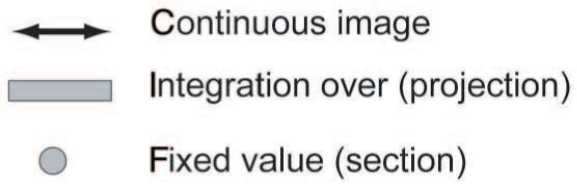

FIGURE 6 The imaging conditions of the three techniques $(a),(b)$ and (c) in the six-dimensional orientation-location space: (1) continuously imaged coordinates; (2) fixed (but selectable) coordinates; (3) not observable coordinates (projection directions). 


\subsection{Orientation Tomography}

The non-measurable angle $\psi$ around the diffraction vector can be obtained by combining several images of type $(a)$.

\subsubsection{ODF Analysis}

In the case of continuous textures, determination of the angle $\psi$ is the well-known ODF analysis, i.e. the calculation of the orientation distribution function $f(\mathbf{g})$ from several pole figures (Bunge, 1982).

\subsubsection{Multipeak Texture Analysis}

In the case of grain-resolved textures the analogues problem requires to find two or more diffraction spots in one (or more) diagrams of type (a), right side, which belong to the same crystallite. A mathematical procedure to solve this problem, called "Multipeak Texture Analysis", was given earlier (Bunge et al., 1989).

It was estimated how many crystallites can be resolved simultaneously, this way, depending on the orientation resolution, i.e. on the accuracy of the coordinates $\{\omega \gamma\}$ in the diagrams of type $(a)$. This is shown in Fig. 7. One sees that the high orientation resolution of synchrotron radiation allows to resolve $\sim 10^{5}$ grains compared with $\sim 10^{2}$ which can be resolved with conventional X-ray diffraction. This is a very strong reason for the use of synchrotron radiation.

\subsection{Determination of the Orientation Stereology}

In diagrams of type $(b)$ the coordinate $x$ of the reflecting volume element, i.e. along the incident beam, cannot be distinguished. Diagrams of type $(b)$ can, however, be taken in

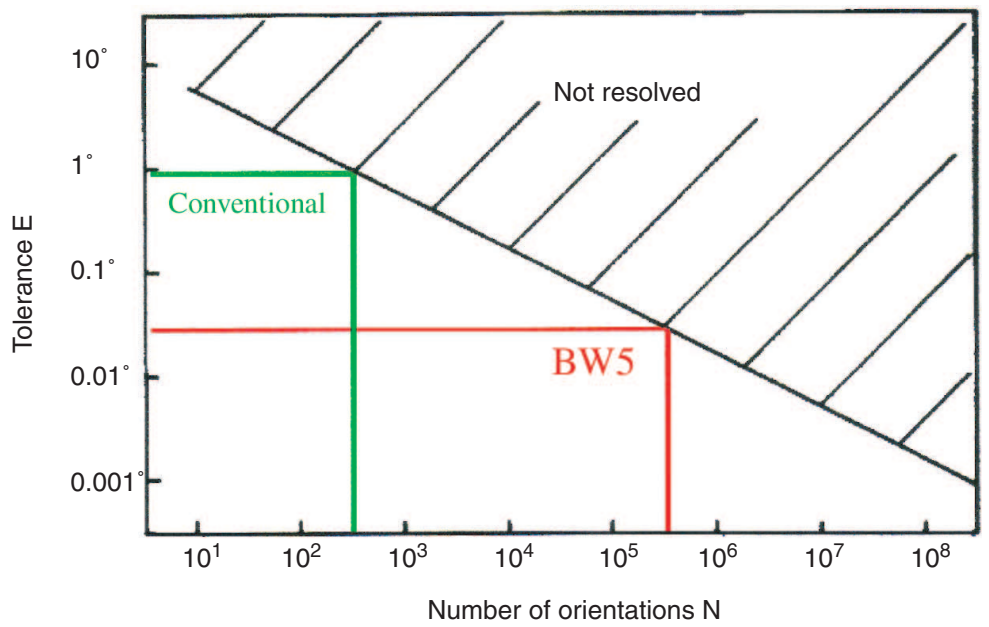

FIGURE 7 Number of grains the orientations of which can be resolved with the "Multipeak" algorithm, green: conventional X-rays, red: synchrotron radiation. 


\section{a)}

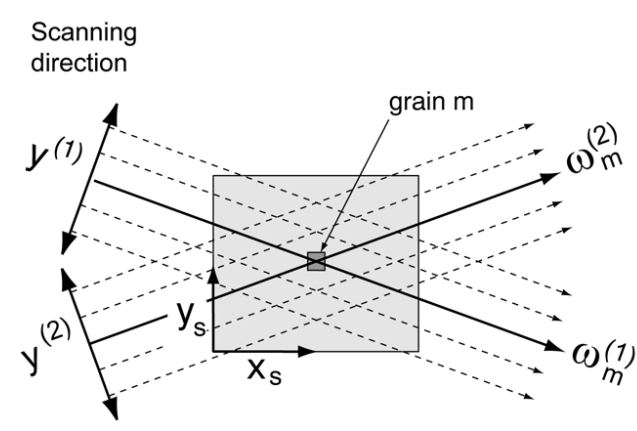

b)

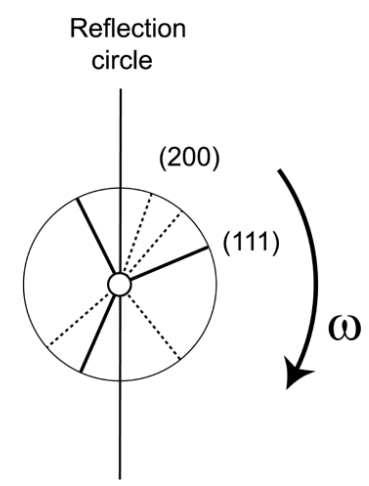

FIGURE 8 Determination of the position $x_{s} y_{s}$ of a reflecting grain in the sample with the technique $(b)$ : (a) the sample is scanned in the goniometer direction $y$ with the sample in two different orientations $\omega_{m}^{1}$ and $\omega_{m}^{2}$ in which the grain $m$ is in reflection orientation; (b) considering (111) and (200) reflections in cubic crystals, each grain $m$ may be seen in a total of $n=7$ orientations $\omega_{m}^{n}$.

different directions of the incident beam with respect to the sample coordinate system. Then the positions of individual grains can be localized at the intersection of these beams, Fig. 8. Thereby, it must be kept in mind that a grain can be "seen" in a diagram only when it is in reflection orientation. If, in the case of cubic crystals, we consider (111) and (200) reflections, then we have the chance to see any grain, number $m$, at seven different sample orientations $\omega_{m n}$. This way, all three position coordinates $\left\{\begin{array}{lll}x_{1} & x_{2} & x_{3}\end{array}\right\}_{m}$ of the grain number $m$ can be obtained when its orientation parameters $\{\omega \gamma \psi\}_{m}$ are known from the method mentioned in the preceding section. Hence, the positions and orientations $\left\{\omega \gamma \psi x_{1} x_{2} x_{3}\right\}_{m}$ of up to $\sim 10^{5}$ grains of a polycrystalline material can be determined simultaneously.

The positions of neighbouring grains determine (approximately) the size and shape of any grain. Hence, the six coordinates $\left\{\omega \gamma \psi x_{1} x_{2} x_{3}\right\}_{m}$ of all grains describe the "orientation stereology" of the polycrystalline aggregate (Bunge and Schwarzer, 2001). The reliability of the solution can be checked by the streak lengths in the diagrams of type $(b)$ which are the grain diameters in the respective scanning directions. If we use all seven theoretically possible reflection orientations (as was mentioned above) then these are seven grain diameters in seven different directions in the $x-y$ plane which describes the cross-section of the grain in this plane fairly well.

\section{COMPARISON WITH FILM TEXTURE GONIOMETER TECHNIQUES}

The principle of "sweeping" a Debye-Scherrer ring (with varying intensity distribution) over an area detector, illustrated in Fig. 2, is not new. It has been used long time ago with photographic film as an area detector (Kratky, 1930; Barrett, 1931; Guinier and Tennevin, 1948). A survey of these techniques was given by Wassermann and Grewen (1962). As an example, Fig. 9 shows the (111) pole figure of a cold rolled 


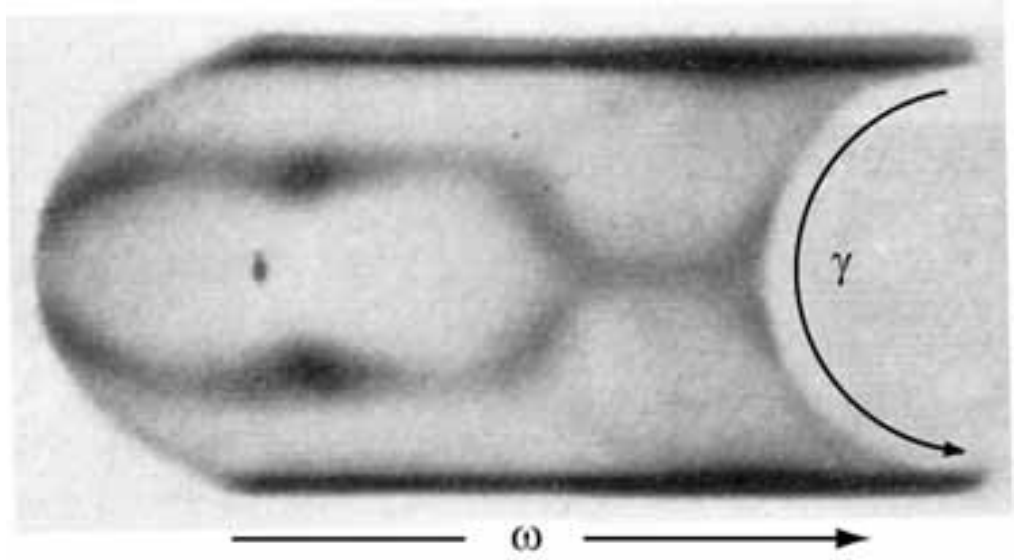

FIGURE 9 (111) pole figure of a cold rolled aluminium sheet obtained with a film texture goniometer (Guinier and von Eller, 1957).

aluminium sheet measured with a film texture goniometer (Guinier and von Eller, 1957). It is to be compared with the diagram Fig. 5(a), left.

\subsection{Rotation of Individual Grains in the Polycrystal}

Also individual grain resolution can be reached with this technique. Figure 10 shows an image of an area of a pole figure of a recrystallized Al-wire obtained with a similar film technique (Bunge, 1967). One sees spots corresponding to $\langle 111\rangle$ directions of individual grains. A second measurement was then done with the same wire after $1.4 \%$ plastic extension (Bunge and Fuchs, 1969). After that, the spots of the individual grains can still be identified but they have moved a little bit according to grain rotation. This allows to measure two (of the three) rotation components of many individual grains, Fig. 11.

The rotation of crystal orientations by plastic deformation can be calculated by different model assumptions. In the Taylor model (Taylor, 1938), for example, it is assumed that each grain undergoes the same deformation. Crystal rotation deduced from this model is, however, not a unique function of the starting orientation. Hence, only the average of the possible rotations can be considered to be relevant in a polycrystalline material. This non-unicity of the Taylor solution is superimposed with the non-unicity of deformation of the individual grains (beyond the Taylor assumption). Finally, the spots in Figs. 10 and 11 specify only two of the three orientation coordinates of the starting crystal orientation. Hence, comparison with the Taylor model (Bunge, 1970) can only be done as an average over very many crystallites. This is shown in Fig. 12 as an average of $\sim 2000$ crystallites.

In Fig. 11 only two of three components of the starting crystal orientation as well as of the rotation could be measured. Furthermore, nothing was known about the local environment of each grain. Using the synchrotron techniques $(a),(b)$ and $(c)$, described in this article, it will, in principle, be possible to measure the complete starting orientations, the environments, the local deformations, and the complete rotations of up to $10^{5}$ crystallites simultaneously. Then much better comparison with the different mathematical models of plastic deformation of polycrystalline materials (see e.g. Bunge, 2000) 


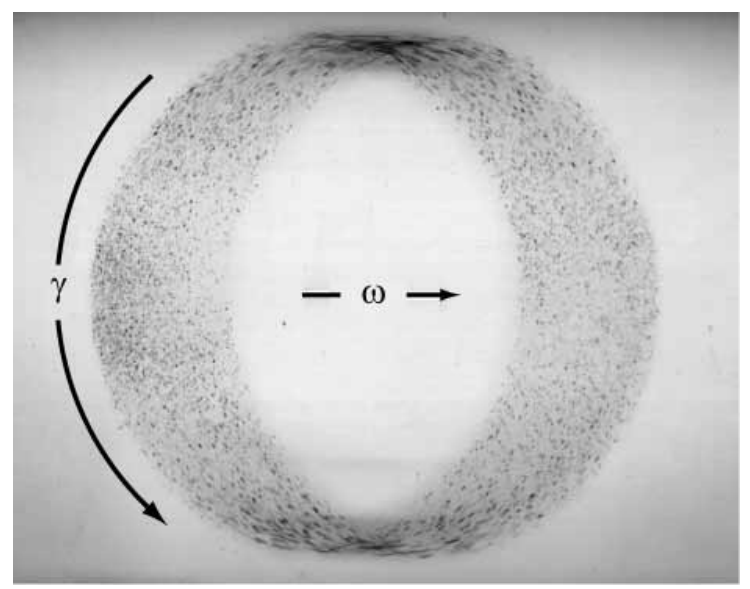

FIGURE 10 Two areas of the (111) pole figure of a recrystallized aluminium wire obtained with a "rotating film" technique (Bunge, 1967).
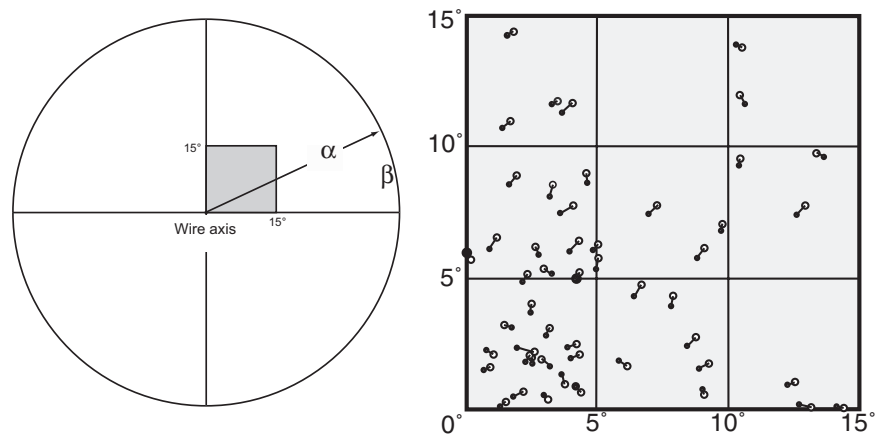

FIGURE 11 The (111) poles from Fig. 10 plotted in the pole figure coordinates $\{\alpha \beta\}: \bigcirc$ before plastic deformation; after $1.4 \%$ extension in axis direction.

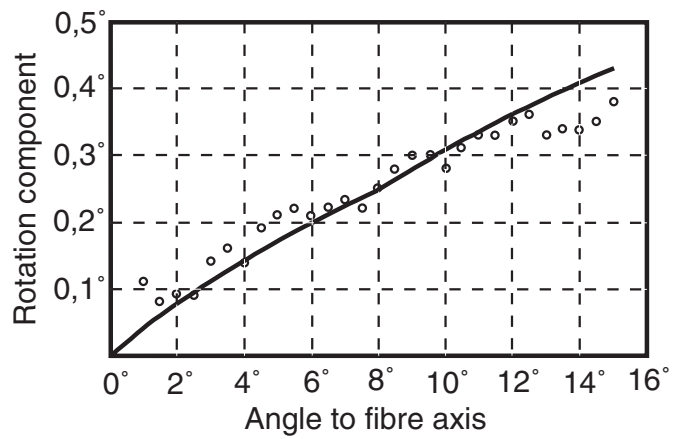

FIGURE 12 Mean values $\langle\Delta \alpha\rangle$ of the rotation components $\Delta \alpha$ as a function of $\alpha$ after $\sim 1 \%$ elongation. The values were obtained as the average of eight measurements ( 2000 grains). The solid curve was obtained from the Taylor theory (Bunge and Fuchs, 1969). 
should be possible. This is, however, still an ambitious project (see e.g. Margulies et al., 2001).

\section{FURTHER APPLICATIONS OF THE IMAGING METHOD}

The three techniques described in Section 2 may have very many applications in basic research as well as in industrial problems of polycrystalline materials of any kind. They can be used for continuous as well as for grain-resolved textures and microstructures as shown in Fig. 5.

In the following, some examples will be given which illustrate the great potential of this technique.

\subsection{Nucleation of New Grains During Recrystallization}

Figure 13 shows the (111) pole figure of a nickel sheet cold rolled $93 \%$ and subsequently annealed $2 \mathrm{~min}$ at $300^{\circ} \mathrm{C}$. This latter treatment is not sufficient for complete

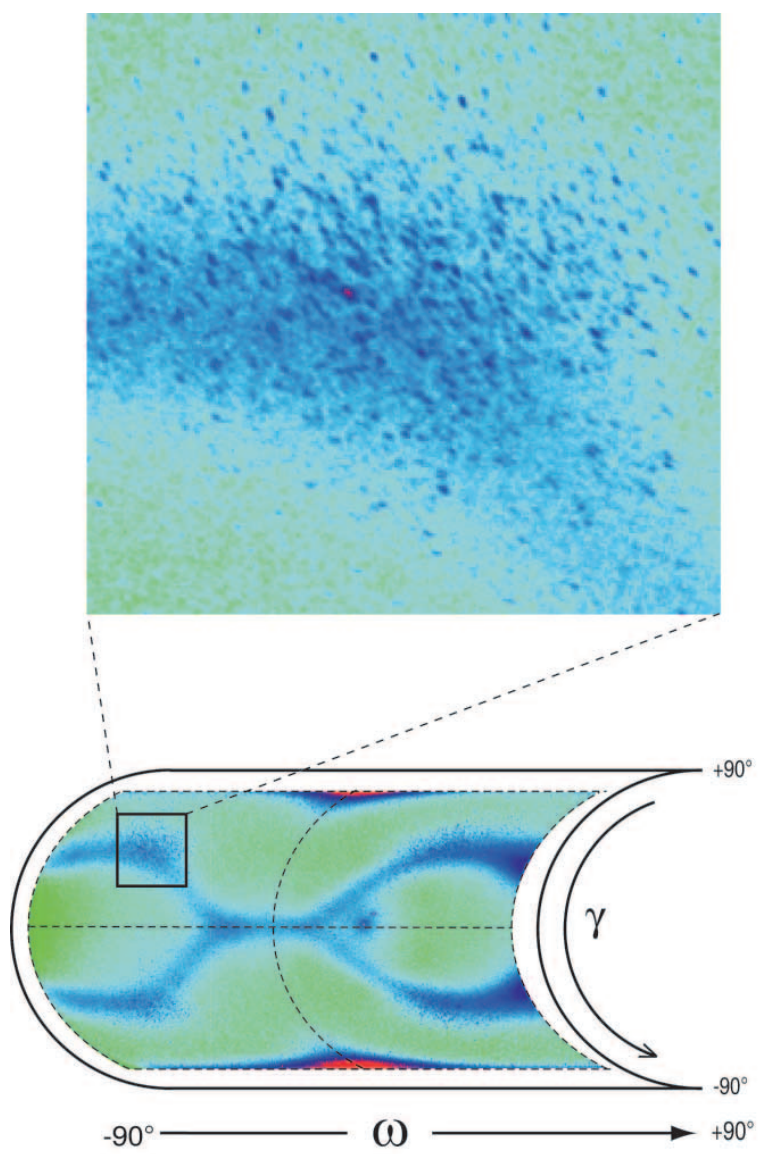

FIGURE 13 (111) pole figure (technique $(a)$ ) of nickel, cold rolled $93 \%$ and annealed 2 min at $300^{\circ} \mathrm{C}$ (partly recrystallized). The enlarged area shows particularly the newly nucleated grains, additionally to the still present deformed material which is better seen in the original scale. 
recrystallization. Thus, one sees spots of newly formed grains besides the continuous intensity distribution of the still present non-recrystallized material. Measurements of this kind can be done in steps throughout the whole recrystallization process. This allows to separate the orientation dependences of nucleation and grain growth during the whole recrystallization process (to be published elsewhere).

\subsection{Texture and Microstructure of Hot Rolled Aluminium}

Figure 14 shows the (200) pole figure of an Al-sample hot rolled $62 \%$ at $490-460^{\circ} \mathrm{C}$. The texture is similar to the recrystallization texture of nickel as shown by Bunge et al. (2003a), i.e. it is essentially the cube texture. However, the cube peaks are strongly elongated in the sense of a rotation around the rolling direction. One also sees that there are recrystallized grains all over the whole pole figure. There are virtually no regions of the pole figure without recrystallized grains. Figure 14 also shows an enlarged area of the pole figure. Here, one can see spots of grains arranged in "garlands". This effect is not observed in materials recrystallized "statically", i.e. without simultaneous plastic deformation. These "garlands" may be attributed to grains nucleated in some orientation correlation to each other.

Figure 15 shows a diagram of type $(b)$ of the same sample. It shows sharp streaks corresponding to well-recrystallized grains besides diffuse spots, clearly distinguished from the sharp streaks. It is assumed that these spots belong to grains which nucleated

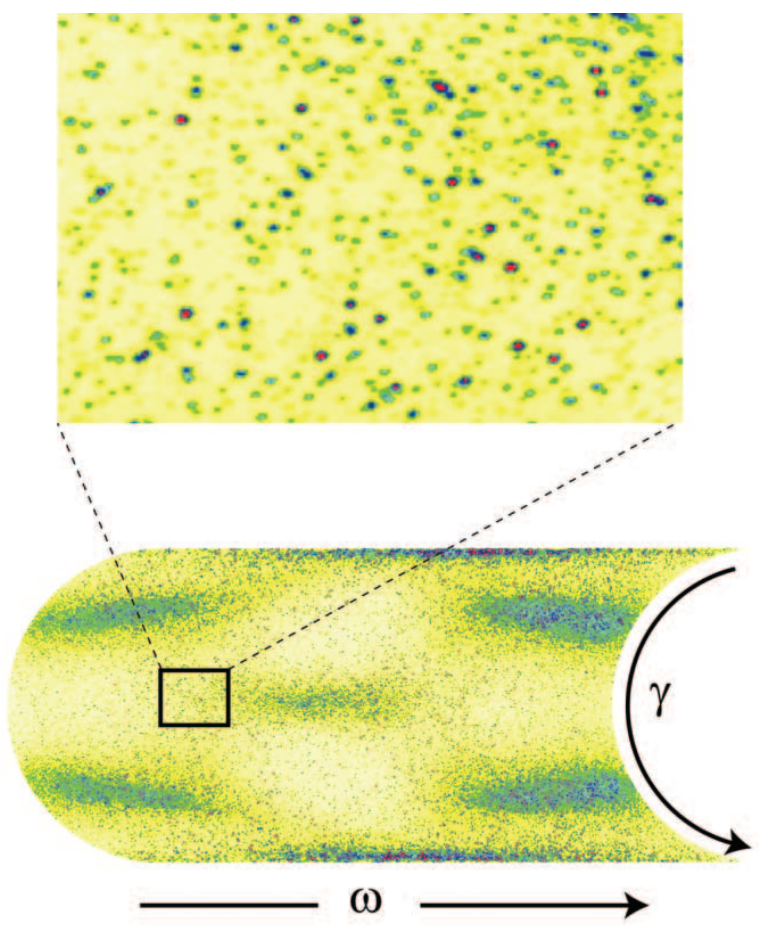

FIGURE 14 (200) pole figure (technique $(a)$ ) of a hot rolled aluminium sample as well as an enlarged area showing particularly the "garland" arrangement of grain orientations. 


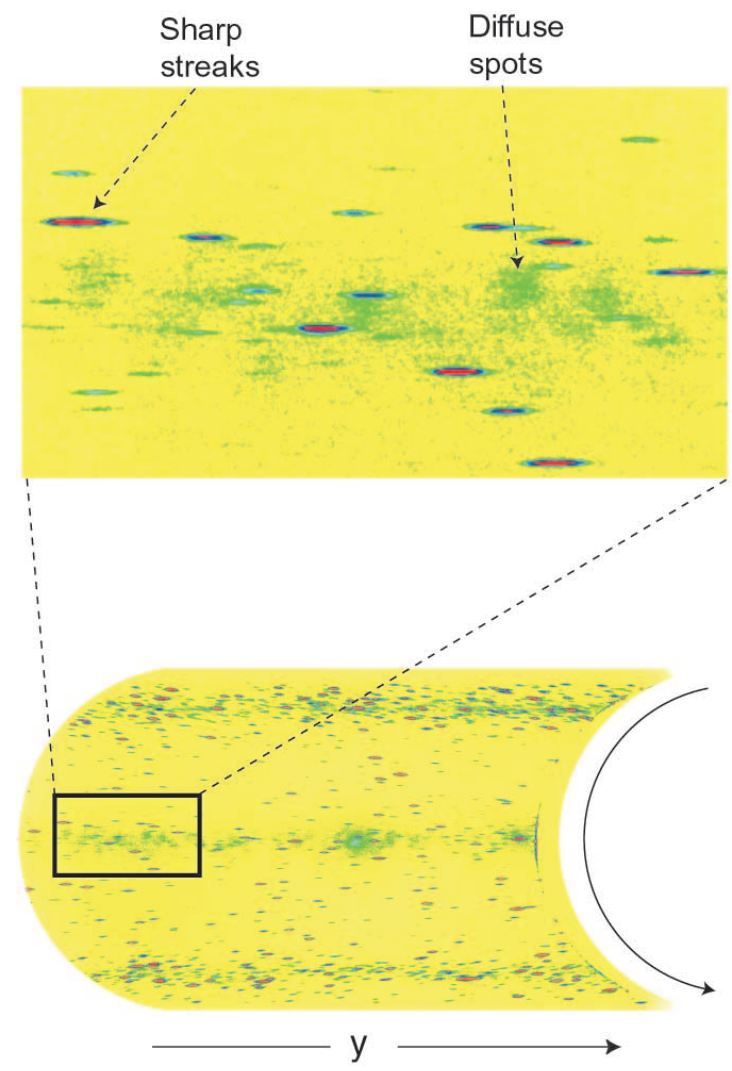

FIGURE 15 (111) scanning diagram (technique $(b)$ ) of the same hot rolled aluminium sample as in Fig. 14. One sees sharp streaks corresponding to crystallites with undistorted crystal lattices, as well as diffuse spots corresponding to crystallites with lattice distortions.

earlier during hot rolling and then underwent some degree of plastic deformation by which they were broadened. Hence, it may be concluded that the hot rolled sample was dynamically recrystallized (details to be published elsewhere).

\subsection{Orientation Relationship of Crystals in the $\gamma \rightarrow \alpha$ Transformation of Iron}

Figure 16(a) shows the (110) pole figure of an iron-nickel meteorite of the Gibeon shower (Buchwald, 1975). It is assumed that the material cooled down in space with a very low cooling rate from a $\gamma$ monocrystal whereby it transformed to a distribution of $\alpha$ crystals with orientation relationships according to the Nishiyama-Wassermann and the Kurdjumov-Sachs model (Weiss and Bunge, 2001; Bunge et al., 2003b). Figure 16(b) shows a calculated pole figure under the assumption that the whole range of orientations between the NW and KS orientations is present. These two orientations are only $5.23^{\circ}$ apart from each other, and the KS orientation has two variants right and left from the NW orientation, as is shown in Fig. 17. One sees that the closestpacked planes of the two crystal lattices, i.e. $(111)_{\gamma}$ and $(110)_{\alpha}$ are parallel within $\pm 1.5^{\circ}$. However, the parallelity of directions in the two planes has a variation range of $10.5^{\circ}$ which has rather sharp boundaries on both sides as is also illustrated in Fig. 17. 
a)
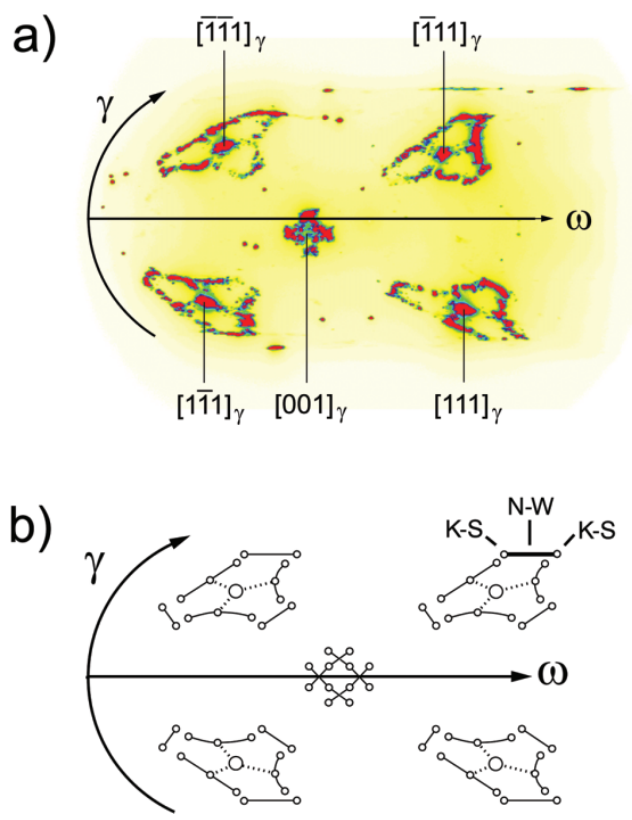

FIGURE 16 (110) pole figure of an iron-nickel meteorite obtained with technique (a): (a) the experimental pole figure; (b) theoretical pole figure corresponding to the assumption of a continuous spread between the Nishiyama-Wassermann and Kurdjumov-Sachs orientation relationship.

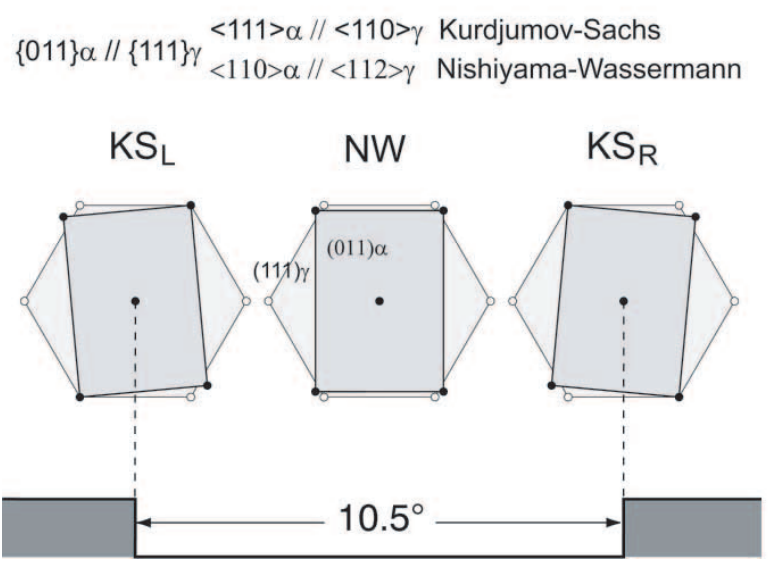

FIGURE 17 The closest-packed lattice planes of $\alpha$ and $\gamma$ iron according to the orientation relationships of Nishiyama-Wassermann and Kurdjumov-Sachs. The "in-plane" orientation relationship may vary in the whole range $\mathrm{KS}_{\mathrm{L}}-\mathrm{NW}-\mathrm{KS}_{\mathrm{R}}$, but it does not exceed this range.

\subsection{Diffraction Image of a Soldering Seam}

Figure 18 shows an iron sheet, the two halves of which were fit together by soldering with a brass-type soldering alloy. The seam was scanned with the technique $(c)$ parallel and perpendicular to it as indicated. The two diffraction images consist of the 


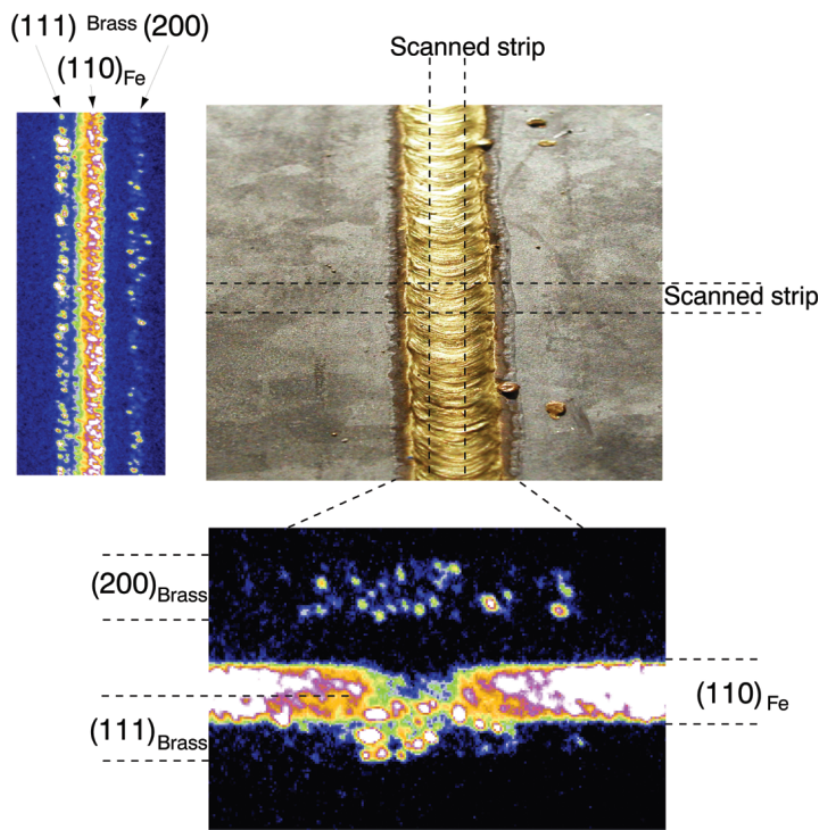

FIGURE 18 Two halves of an iron sheet connected by a soldering seam of a brass-type alloy. The sample was scanned along the seam and perpendicular to it with the technique $(c)$. The diffraction images show the $(110)_{\mathrm{Fe}}$ as well as $(111)_{\text {brass }}$ and $(200)_{\text {brass }}$ reflections (Note that the three partial figures are not in the same scale).

reflections $(110)_{\mathrm{Fe}}$ as well as $(111)_{\text {brass }}$ and $(200)_{\text {brass }}$. The scans of the first two reflections are overlapped as a consequence of the slit height of $4 \mathrm{~mm}$ in $z$-direction, the third one is well separated. In the perpendicular scan one sees the diffraction from the iron sheet which is interrupted in the middle and is modified in the heat-influenced zone. The two brass reflection clearly reveal two different texture components in the soldering seam. The texture component seen in the $(111)_{\text {brass }}$ reflection is concentrated in the middle of the seam whereas that in the $(200)_{\text {brass }}$ reflection is extended over the whole seam. The scan parallel to the seam is virtually homogeneous within the statistical limits according to grain size.

\subsection{Texture in the Wall of a Nivea Box}

A nivea box, deep drawn out of an Al sheet, was scanned along its circumference as is shown schematically in Fig. 19(a). In this case, rotation around the axis $\omega$ corresponds to a translation along the circumference of the box. Hence, this technique may be considered as a variant of technique $(b)$. The diameter of $11 \mathrm{~cm}$ of the box allows easily to separate the reflections from the front and rear part of the cylindrical wall by the diffraction angle slit. Figure 19(b) shows the diffraction image in the light of the (111) reflection. One sees a strong inhomogeneity of the texture along the circumference. The exact production process of the box was not known, but it may be assumed that it was deep drawn from a textured sheet, presumably with a strong cube texture. Thereby the radial directions of the blank become parallel to the axis direction of the box. However, each part of the wall is deformed differently with respect to the texture 

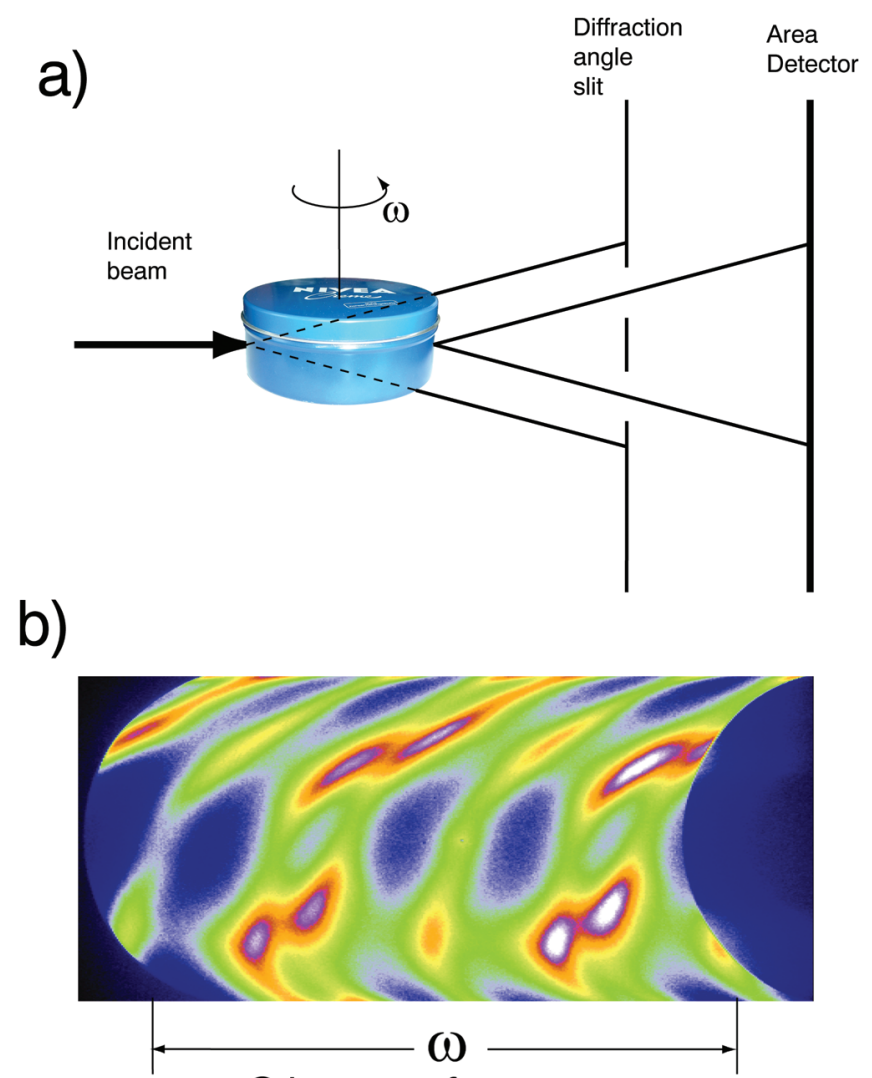

Circumference

FIGURE 19 (a) A Nivea box scanned along its circumference with the diffraction angle slit and moving detector. With respect to the "unrolled" wall of the box this corresponds to technique (b). (b) Diffraction image, technique (b), scanned along the circumference of the box.

directions in the initial blank. Hence, Fig. 19 is a fast inspection of the texture but not a complete description of it.

\section{INFLUENCE OF MICROSTRUCTURE ON THE MACROSCOPIC PROPERTIES OF A MATERIAL}

The macroscopic physical properties of a polycrystalline material are the orientation averages of the anisotropic properties of its crystallites. Thereby the orientation distribution function (ODF) is the weighting function (Bunge, 1982). In the case of the elastic properties, two different model assumptions are usually made for that, i.e. the models according to Voigt (1928) (constant strain) or Reuss (1929) (constant stress). The deviation between the two increases with increasing anisotropy of the crystallites. The Voigt model was developed from a microstructure consisting of long parallel (pencil-shaped) crystallites, whereas the Reuss-model corresponds to a stack of flat (pancake-shaped) crystallites. Hence, it is obvious that the actual grain shape and neighbourhood arrangement of the crystallites must have an influence on the macroscopic properties 


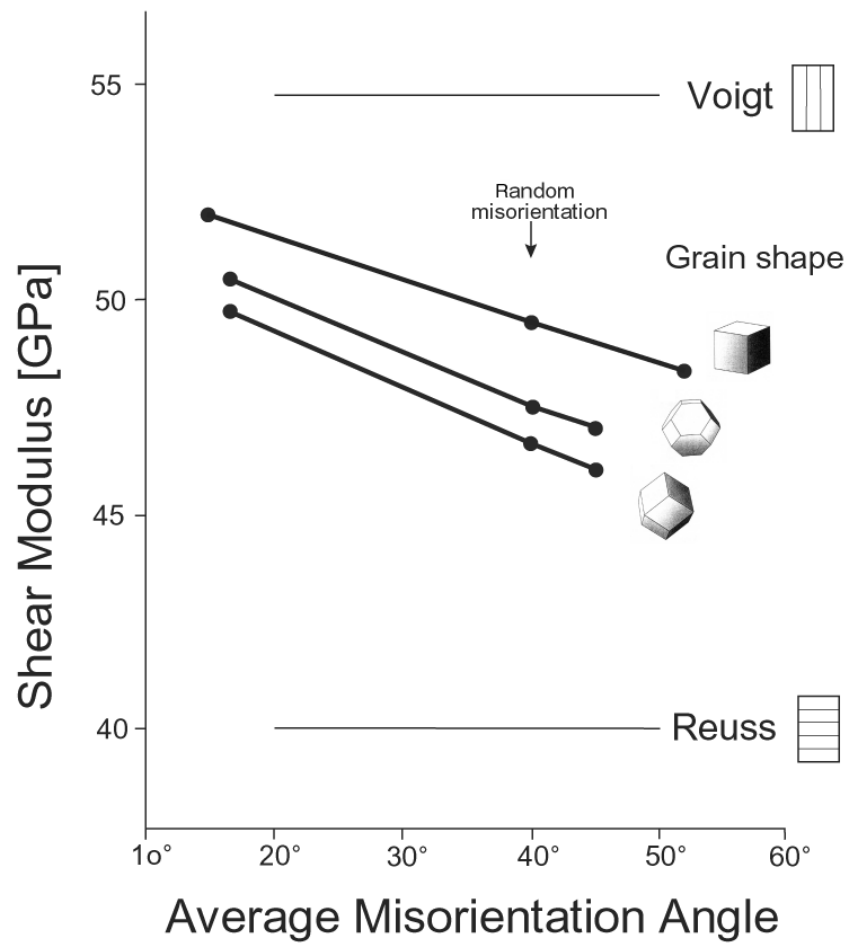

FIGURE 20 Influence of two particular microstructural parameters, i.e. grain shape and average misorientation angle, on the elastic properties (i.e. the shear modulus) of copper (with random texture). The values vary within the Voigt-Reuss bounds.

within the Voigt-Reuss intervall. Figure 20 shows model calculations of the elastic shear modulus of copper with random orientation distribution for different assumed microstruture parameters. The calculations were done with the cluster model (Bunge et al., 2000). One sees that the used parameters result in macroscopic properties filling already an essential part of the Voigt-Reuss interval. Hence, it is necessary to measure the complete orientation stereology of a polycrystalline material, e.g. with the techniques $(a),(b)$ and $(c)$ described in Section 2 and then to calculate the macroscopic properties on this basis. This is one of the projects to be done in the future.

\section{SUMMARY AND CONCLUSIONS}

Diffraction imaging with hard (short-wave) synchrotron radiation, using the detector sweeping techniques described in this article allows to measure the texture and microstructure of polycrystalline materials with high orientation- and location-resolution. These techniques, summarized in Fig. 5, provide continuous two-dimensional images of different sections and projections of the six-dimensional "orientation-location" space. These images must be distinguished according to whether individual grains can be resolved in them or not. In the first case (the right side of Fig. 5) it is thus possible to measure the orientation and location coordinates $\left\{\varphi_{1} \Phi \varphi_{2} x_{1} x_{2} x_{3}\right\}_{m}$ of up to $10^{5}$ individual grains simultaneously. From these parameters also grain size and shape 
can be obtained (at least in good approximation). This is the complete orientation stereology of the polycrystalline aggregate. In the second case (the left side of Fig. 5) the intensity in any point of the diagrams corresponds to a pole density as a function of $\{\omega \gamma y z\}$. Thereby, it is always projected (or integrated) over the angle $\psi$ around the diffraction vector and the coordinate $x$ in beam direction.

An essential feature of the methods described here is their orientation- and locationresolving power. The first one depends on the the divergence of the incident beam and the spectral width of the monochromator. The second one depends on the size of the entrance slit combined with the intensity of the primary beam which allows a sufficient intensity at the sample even with a very narrow beam. It must be mentioned here that, in the examples given in the present article, both resolving powers were not yet at their physical limits. Hence, it is intended, in the future work, to increase both, orientation- as well as location-resolution.

Diffraction imaging with high-energy synchrotron radiation must be compared with location scanning of electron diffraction (Adams et al., 1994; Schwarzer, 1997). Both these techniques are complementary to each other in different respects. High-energy synchrotron radiation is capable of reaching higher orientation resolution, whereas electron diffraction may reach higher lateral resolution. Besides that, electron diffraction "sees" only a very thin (two-dimensional) layer, i.e. either the surface of the sample in reflection or a thinned sample in transmission, whereas high-energy synchrotron radiation can reveal texture and microstructure in the bulk of big or even encapsulated samples. Also, individual structural components inside complex technological units can be investigated non-destructively.

Hence, the described methods are particularly suited for the study of the behaviour of individual crystallites inside polycrystalline materials, e.g., orientation changes during plastic deformation, or volume changes during recrystallization, grain growth, or phase transformation. Furthermore, they are indispensible for the understanding of macroscopic physical properties of materials with accuracies better than the Voigt-Reuss uncertainty as was shown in Fig. 20.

\section{References}

Adams, B.L.,Dingley, D.J., Kunze, K. and Wright, S.L. (1994). Orientation imaging microscopy: new possibilities for microstructural investigations using automated BKD analysis. Mater. Sci. Forum, 157-162, 31-42.

Barrett, C.S. (1931). Trans AIME, 93, 75-77.

Buchwald, V.F. (1975). Handbook of Iron Meteorites. Univ. of California Press, Berkeley.

Bunge, H.J. (1967). Eine röntgenographische Methode zur Messung der Orientierungsabhängigkeit der Korngröße. Z. Metallkde., 58, 649-657.

Bunge, H.J. (1970). Some applications of Taylor‘s theory of polycrystal plasticity. Kristall u. Techn., 5, $145-175$.

Bunge, H.J. (1982). Texture Analysis in Materials Science, Butterworths Publ., London; 2nd Edn., Cuvillier Verlag Göttingen, 1993.

Bunge, H.J. (2000). Crystallographic texture and plastic anisotropy. In: Banabic, D. (Ed.), Formability of Metallic Materials. Springer Verlag, Berlin.

Bunge, H.J. and Fuchs, R. (1969). On the orientation changes during plastic deformation of individual crystallites in polycrystalline aggregates. Phys. Stat. Sol., 32, 169-177.

Bunge, H.J., Kiewel, R., Reinert, T. and Fritsche, L. (2000). Elastic properties of polycrystals - influence of texture and stereology. J. Mech. Phys. Sol., 48, 29-66.

Bunge, H.J., Morris, P. and Nauer-Gerhardt, C.U. (1989). ODF-analysis of multipeak textures. Textures and Microstructures, 11, 1-22.

Bunge, H.J. and Schwarzer, R.A. (2001). Orientation stereology - a new branch in texture research. Advanced Engin. Mater., 3, 25-39. 
Bunge, H.J., Wcislak, L., Klein, H., Garbe, U. and Schneider, J.R. (2002). Texture and microstructure analysis with high-energy synchrotron radiation. Advanced Engin. Mater., 4, 300-305.

Bunge, H.J., Wcislak, L., Klein, H., Garbe, U. and Schneider, J.R. (2003a). Texture and microstructure imaging in six dimensions with high-energy synchrotron radiation. J. Appl.Cryst., 36, 1240-1255.

Bunge, H.J., Weiss, W., Klein, H., Wcislak, L., Garbe, U. and Schneider, J.R. (2003b). Orientation relationship of Widmannstätten plates in an iron meteorite measured with high-energy synchrotron radiation. J. Appl. Cryst., 36, 137-140.

Guinier, A. and von Eller, G. (1957). Les methodes experimentales des determinations de structures crystallines par rayons x. In: Flügge, S. (Ed.), Encyclopedia of Physics, Vol. 32, pp. 1-96. Springer Verlag, Berlin.

Guinier, A. and Tennevin, J. (1948). Rev. Metall., 45, 277-286.

Kratky, O. (1930). Z. Kristallogr., 72, 529-540.

Margulies, L., Winther, G. and Poulsen, H.F. (2001). In-situ measurement of grain rotation during deformation of polycrystals. Science, 292, 2392-2394.

Reuss, A. (1929). Berechnung der Fließgrenze von Mischkristallen auf Grund der Plastizitätsbedingung für Einkristalle. Z. Angew. Math. Mech., 9, 49-58.

Schwarzer, R.A. (1997). Automated crystal lattice orientation mapping using a computer-controlled SEM. Micron, 28, 249-265.

Taylor, G.I. (1938). Plastic strain in metals. J. Inst. Metals, 62, 307-324.

Voigt, W. (1928). Lehrbuch der Kristallphysik. B.G. Teubner Verlag, Leipzig.

Wassermann, G. and Grewen, J. (1962). Texturen metallischer Werkstoffe. Springer Verlag, Berlin.

Wcislak, L., Klein, H., Bunge, H.J., Garbe, U., Tschentscher, T. and Schneider, J.R. (2002). Texture analysis with high-energy synchrotron radiation. J. Appl. Cryst., 35, 82-95.

Weiss, W. and Bunge, H.J. (2001). Diffraction contrast in X-ray radiographic images of octahedrite meteorites. J. Appl. Cryst., 34, 566-572. 